\title{
A Coupled Fixed Point Theorem in Fuzzy Metric Space Satisfying $\phi$-Contractive Condition
}

\author{
B. D. Pant, ${ }^{1}$ Sunny Chauhan, ${ }^{2}$ Jelena Vujaković, ${ }^{3}$ \\ Muhammad Alamgir Khan, ${ }^{4}$ and Calogero Vetro ${ }^{5}$ \\ ${ }^{1}$ Government Degree College, Champawat, Uttarakhand 262523, India \\ ${ }^{2}$ R. H. Government Postgraduate College, Kashipur (Udham Singh Nagar), Uttarakhand 244713, India \\ ${ }^{3}$ Faculty of Sciences and Mathematics, Lole Ribara 29, 38200 Kosovska Mitrovica, Serbia \\ ${ }^{4}$ Department of Natural Resources Engineering and Management, University of Kurdistan, Erbil 22570, Iraq \\ ${ }^{5}$ Università degli Studi di Palermo, Dipartimento di Matematica e Informatica, Via Archirafi 34, 90123 Palermo, Italy
}

Correspondence should be addressed to B. D. Pant; badridatt.pant@gmail.com

Received 4 June 2013; Revised 21 July 2013; Accepted 8 August 2013

Academic Editor: Salvatore Sessa

Copyright (C) 2013 B. D. Pant et al. This is an open access article distributed under the Creative Commons Attribution License, which permits unrestricted use, distribution, and reproduction in any medium, provided the original work is properly cited.

\begin{abstract}
The intent of this paper is to prove a coupled fixed point theorem for two pairs of compatible and subsequentially continuous (alternately subcompatible and reciprocally continuous) mappings, satisfying $\phi$-contractive conditions in a fuzzy metric space. We also furnish some illustrative examples to support our results.
\end{abstract}

\section{Introduction}

The evolution of fuzzy mathematics commenced with the introduction of the notion of fuzzy sets by Zadeh [1], where the concept of uncertainty was introduced in the theory of sets, in a nonprobabilistic manner. Fuzzy set theory has applications in applied sciences such as mathematical programming, model theory, engineering sciences, image processing, and control theory. In 1975, Kramosil and Michalek [2] introduced the concept of fuzzy metric space as a generalization of the statistical (probabilistic) metric space. Afterwards, Grabiec [3] defined the completeness of the fuzzy metric space and extended the Banach contraction principle to fuzzy metric spaces. Since then, many authors contributed to the development of this theory, also in relation to fixed point theory (e.g., [4-9]).

Mishra et al. [10] extended the notion of compatible mappings (introduced by Jungck [11] in metric spaces) to fuzzy metric spaces and proved common fixed point theorems in presence of continuity of at least one of the mappings, completeness of the underlying space, and containment of the ranges amongst involved mappings. Further, Singh and Jain [12] weakened the notion of compatibility by using the notion of weakly compatible, mappings in fuzzy metric spaces and showed that every pair of compatible mappings is weakly compatible but converse is not true. Inspired by Bouhadjera and Godet-Thobie [13, 14], Gopal and Imdad [15] extended the notions of subcompatibility and subsequential continuity to fuzzy metric spaces and proved fixed point theorems using these notions together due to Imdad et al. [16]. In recent past, several authors proved various fixed point theorems employing more general contractive conditions (e.g., [17-26]).

On the other hand, Bhaskar and Lakshmikantham [27] and Lakshmikantham and Ćirić [28] gave some coupled fixed point theorems in partially ordered metric spaces (see also [29-31]). In 2010, Sedghi et al. [32] proved common coupled fixed point theorems in fuzzy metric spaces for commuting mappings. Motivated by the results of [33], $\mathrm{Hu}$ [34] proved a coupled fixed point theorem for compatible mappings satisfying $\phi$-contractive conditions in fuzzy metric spaces with continuous $\mathrm{t}$-norm of $\mathrm{H}$-type and generalized the result of Sedghi et al. [32]. In an interesting note, Zhu and Xiao [35] showed that the results contained in Sedghi et al. [32] are not true in their present form.

Inspired by the work of Zhu and Xiao [35], we prove coupled common fixed point theorems for two pairs of 
mappings satisfying a general contractive condition in fuzzy metric spaces, by using the notions of compatibility and subsequential continuity (alternately subcompatibility and reciprocal continuity). Our results improve many known common coupled fixed point theorems available in the existing literature. We support our results with two illustrative examples.

\section{Preliminaries}

In this section, we collect some basic notions and results. In the sequel $\mathbb{R}^{+}$will denote the set of all positive real numbers while $\mathbb{N}$ will denote the set of natural numbers.

Definition 1 (see [1]). Let $X$ be any set. A fuzzy set $A$ in $X$ is a function with domain $X$ and values in $[0,1]$.

Definition 2 (see [36]). A binary operation $*:[0,1] \times$ $[0,1] \rightarrow[0,1]$ is a continuous $t$-norm if $*$ satisfies the following conditions:

(a) * is commutative and associative;

(b) $*$ is continuous;

(c) $a * 1=a$ for all $a \in[0,1]$;

(d) $a * b \leq c * d$ whenever $a \leq c$ and $b \leq d$ for all $a, b, c, d \in$ $[0,1]$.

Definition 3 (see [37]). One says that a $t$-norm $*$ is of H-type if the family $\left\{*^{n}\right\}$ of its iterates is equicontinuous at $x=1$; that is, for any $\lambda \in(0,1)$, there exists $\delta(\lambda) \in(0,1)$ such that $x>1-\delta$ implies $*^{n}(x)>1-\lambda$, for all $n \in \mathbb{N}$.

The $t$-norm $*_{m}=\min \{a, b\}$ for all $a, b \in[0,1]$ is an example of $t$-norm of $\mathrm{H}$-type, but there are some other $t$ norms $*$ of H-type (see [37]).

Definition 4 (see [2]). A 3-tuple $(X, M, *)$ is said to be a fuzzy metric space if $X$ is an arbitrary nonempty set, $*$ is a continuous $t$-norm, and $M$ is a fuzzy set in $X^{2} \times(0,+\infty)$ satisfying the following conditions, for each $x, y, z \in X$ and $t, s>0$ :
(a) $M(x, y, t)>0$;
(b) $M(x, y, t)=1$ for all $t>0$ if and only if $x=y$;
(c) $M(x, y, t)=M(y, x, t)$;
(d) $M(x, y, t) * M(y, z, s) \leq M(x, z, t+s)$;
(e) $M(x, y, \cdot):(0, \infty) \rightarrow(0,1]$ is continuous.

Example 5 (see [7]). Let $(X, d)$ be a metric space. Define the $t$-norm $a * b=a b$ for all $a, b \in[0,1]$, and, for all $x, y \in X$ and $t>0$,

$$
M(x, y, t)=\frac{t}{t+d(x, y)} .
$$

Then $(X, M, *)$ is a fuzzy metric space, and the fuzzy metric $M$ induced by the metric $d$ is often referred, as the standard fuzzy metric.
Example 6 (see [32]). Let $(X, d)$ be a metric space and $\psi$ be an increasing and continuous function from $\mathbb{R}^{+}$into $(0,1)$ such that $\lim _{s \rightarrow \infty} \psi(s)=1$. Four typical examples of these functions are $\psi(s)=s /(s+1), \psi(s)=\sin (\pi s /(2 s+1))$, $\psi(s)=1-e^{-s}$, and $\psi(s)=e^{-1 / s}$. Let $a * b=a b$ for all $a, b \in[0,1]$, and, for each $x, y \in X$ and $t>0$, define

$$
M(x, y, t)=[\psi(t)]^{d(x, y)} .
$$

It is easy to see that $(X, M, *)$ is a fuzzy metric space.

Definition 7 (see [34]). Define $\Phi=\left\{\phi: \mathbb{R}^{+} \rightarrow \mathbb{R}^{+}\right\}$such that $\phi \in \Phi$ satisfies the following conditions:

$(\phi-1) \phi$ is nondecreasing;

$(\phi-2) \phi$ is upper semicontinuous from the right;

$(\phi-3) \sum_{n=0}^{\infty} \phi^{n}(s)<+\infty$ for all $s>0$, where $\phi^{n+1}(s)=$ $\phi\left(\phi^{n}(s)\right), n \in \mathbb{N}$.

Clearly if $\phi \in \Phi$, then $\phi(s)<s$ for all $s>0$.

Definition 8 (see [27]). An element $(x, y) \in X \times X$ is called

(a) a coupled fixed point of the mapping $f: X \times X \rightarrow X$ if

$$
f(x, y)=x, \quad f(y, x)=y
$$

(b) a coupled coincidence point of the mappings $f: X \times$ $X \rightarrow X$ and $g: X \rightarrow X$ if

$$
f(x, y)=g(x), \quad f(y, x)=g(y) ;
$$

(c) a common coupled fixed point of the mappings $f$ : $X \times X \rightarrow X$ and $g: X \rightarrow X$ if

$$
\begin{aligned}
& x=f(x, y)=g(x), \\
& y=f(y, x)=g(y) .
\end{aligned}
$$

Definition 9 (see [27]). An element $x \in X$ is called a common fixed point of the mappings $f: X \times X \rightarrow X$ and $g: X \rightarrow X$ if

$$
x=g(x)=f(x, x) .
$$

Definition 10 (see [34]). The mappings $f: X \times X \rightarrow X$ and $g: X \rightarrow X$ are called compatible if

$$
\begin{aligned}
& \lim _{n \rightarrow \infty} M\left(g f\left(x_{n}, y_{n}\right), f\left(g x_{n}, g y_{n}\right), t\right)=1, \\
& \lim _{n \rightarrow \infty} M\left(g f\left(y_{n}, x_{n}\right), f\left(g y_{n}, g x_{n}\right), t\right)=1,
\end{aligned}
$$

for all $t>0$, whenever $\left\{x_{n}\right\}$ and $\left\{y_{n}\right\}$ are sequences in $X$ such that

$$
\begin{aligned}
& \lim _{n \rightarrow \infty} f\left(x_{n}, y_{n}\right)=\lim _{n \rightarrow \infty} g x_{n}=\alpha, \\
& \lim _{n \rightarrow \infty} f\left(y_{n}, x_{n}\right)=\lim _{n \rightarrow \infty} g y_{n}=\beta,
\end{aligned}
$$

for some $\alpha, \beta \in X$. 
Now we introduce the following notions.

Definition 11. The mappings $f: X \times X \rightarrow X$ and $g: X \rightarrow X$ are said to be reciprocally continuous if, for sequences $\left\{x_{n}\right\}$, $\left\{y_{n}\right\}$ in $X$, one has

$$
\begin{gathered}
\lim _{n \rightarrow \infty} f\left(g x_{n}, g y_{n}\right)=f(\alpha, \beta), \\
\lim _{n \rightarrow \infty} g f\left(x_{n}, y_{n}\right)=g \alpha, \\
\lim _{n \rightarrow \infty} f\left(g y_{n}, g x_{n}\right)=f(\beta, \alpha), \\
\lim _{n \rightarrow \infty} g f\left(y_{n}, x_{n}\right)=g \beta,
\end{gathered}
$$

whenever

$$
\begin{aligned}
& \lim _{n \rightarrow \infty} f\left(x_{n}, y_{n}\right)=\lim _{n \rightarrow \infty} g x_{n}=\alpha, \\
& \lim _{n \rightarrow \infty} f\left(y_{n}, x_{n}\right)=\lim _{n \rightarrow \infty} g y_{n}=\beta,
\end{aligned}
$$

for some $\alpha, \beta \in X$.

If two self-mappings are continuous, then they are obviously reciprocally continuous, but the converse is not true. Moreover, in the setting of common fixed point theorems for compatible pairs of self mappings satisfying contractive conditions, continuity of one of the mappings implies their reciprocal continuity but not conversely (see [38]).

Definition 12. The mappings $f: X \times X \rightarrow X$ and $g: X \rightarrow X$ are said to be subsequentially continuous if and only if there exist sequences $\left\{x_{n}\right\},\left\{y_{n}\right\}$ in $X$ such that

$$
\begin{aligned}
& \lim _{n \rightarrow \infty} f\left(x_{n}, y_{n}\right)=\lim _{n \rightarrow \infty} g x_{n}=\alpha, \\
& \lim _{n \rightarrow \infty} f\left(y_{n}, x_{n}\right)=\lim _{n \rightarrow \infty} g y_{n}=\beta,
\end{aligned}
$$

for some $\alpha, \beta \in X$, and

$$
\begin{gathered}
\lim _{n \rightarrow \infty} f\left(g x_{n}, g y_{n}\right)=f(\alpha, \beta), \\
\lim _{n \rightarrow \infty} g f\left(x_{n}, y_{n}\right)=g \alpha, \\
\lim _{n \rightarrow \infty} f\left(g y_{n}, g x_{n}\right)=f(\beta, \alpha), \\
\lim _{n \rightarrow \infty} g f\left(y_{n}, x_{n}\right)=g \beta .
\end{gathered}
$$

One can easily check that if two self mappings $f$ and $g$ are both continuous, hence also reciprocally continuous mappings but $f$ and $g$ are not sub-sequentially continuous (see [38, Example 1]).

Definition 13. The mappings $f: X \times X \rightarrow X$ and $g$ : $X \rightarrow X$ are said to be subcompatible if and only if there exist sequences $\left\{x_{n}\right\},\left\{y_{n}\right\}$ in $X$ such that

$$
\begin{aligned}
& \lim _{n \rightarrow \infty} f\left(x_{n}, y_{n}\right)=\lim _{n \rightarrow \infty} g x_{n}=\alpha, \\
& \lim _{n \rightarrow \infty} f\left(y_{n}, x_{n}\right)=\lim _{n \rightarrow \infty} g y_{n}=\beta,
\end{aligned}
$$

for some $\alpha, \beta \in X$, and

$$
\begin{aligned}
& \lim _{n \rightarrow \infty} M\left(f\left(g x_{n}, g y_{n}\right), g f\left(x_{n}, y_{n}\right), t\right)=1, \\
& \lim _{n \rightarrow \infty} M\left(f\left(g y_{n}, g x_{n}\right), g f\left(y_{n}, x_{n}\right), t\right)=1,
\end{aligned}
$$

for all $t>0$.

\section{Results}

In this section, we state and prove our fixed point results.

Theorem 14. Let $(X, M, *)$ be a fuzzy metric space, where * is a continuous $t$-norm of $H$-type such that $M(x, y, t) \rightarrow 1$ as $t \rightarrow \infty$, for all $x, y \in X$. Let $A, B: X \times X \rightarrow X$ and $S, T: X \rightarrow X$ be four mappings such that

(a) the pairs $(A, S)$ and $(B, T)$ are compatible and subsequentially continuous;

(b) there exists $\phi \in \Phi$ such that

$$
\begin{aligned}
& M(A(x, y), B(u, v), \phi(t)) \\
& \quad \geq M(S x, T u, t) * M(S y, T v, t),
\end{aligned}
$$

for all $x, y, u, v \in X$ and $t>0$.

Then there exists a unique point $\alpha$ in $X$ such that $\alpha=S \alpha=$ $T \alpha=A(\alpha, \alpha)=B(\alpha, \alpha)$.

Proof. Since the mappings $A$ and $S$ are subsequentially continuous and compatible, there exist sequences $\left\{x_{n}\right\},\left\{y_{n}\right\}$ in $X$ such that

$$
\begin{aligned}
& \lim _{n \rightarrow \infty} A\left(x_{n}, y_{n}\right)=\lim _{n \rightarrow \infty} S x_{n}=\alpha, \\
& \lim _{n \rightarrow \infty} A\left(y_{n}, x_{n}\right)=\lim _{n \rightarrow \infty} S y_{n}=\beta,
\end{aligned}
$$

for all $\alpha, \beta \in X$, and

$$
\begin{aligned}
& \lim _{n \rightarrow \infty} M\left(A\left(S x_{n}, S y_{n}\right), S A\left(x_{n}, y_{n}\right), t\right)=1, \\
& \lim _{n \rightarrow \infty} M\left(A\left(S y_{n}, S x_{n}\right), S A\left(y_{n}, x_{n}\right), t\right)=1,
\end{aligned}
$$

that is $A(\alpha, \beta)=S \alpha$ and $A(\beta, \alpha)=S \beta$. Similarly, with respect to the pair $(B, T)$, there exist sequences $\left\{x_{n}^{\prime}\right\},\left\{y_{n}^{\prime}\right\}$ in $X$ such that

$$
\begin{aligned}
& \lim _{n \rightarrow \infty} B\left(x_{n}^{\prime}, y_{n}^{\prime}\right)=\lim _{n \rightarrow \infty} T x_{n}^{\prime}=\alpha^{\prime}, \\
& \lim _{n \rightarrow \infty} B\left(y_{n}^{\prime}, x_{n}^{\prime}\right)=\lim _{n \rightarrow \infty} T y_{n}^{\prime}=\beta^{\prime},
\end{aligned}
$$

for all $\alpha^{\prime}, \beta^{\prime} \in X$, and

$$
\begin{aligned}
& \lim _{n \rightarrow \infty} M\left(B\left(T x_{n}^{\prime}, T y_{n}^{\prime}\right), T B\left(x_{n}^{\prime}, y_{n}^{\prime}\right), t\right)=1, \\
& \lim _{n \rightarrow \infty} M\left(B\left(T y_{n}^{\prime}, T x_{n}^{\prime}\right), T B\left(y_{n}^{\prime}, x_{n}^{\prime}\right), t\right)=1,
\end{aligned}
$$

that is $B\left(\alpha^{\prime}, \beta^{\prime}\right)=T \alpha^{\prime}$ and $B\left(\beta^{\prime}, \alpha^{\prime}\right)=T \beta^{\prime}$. Hence $(\alpha, \beta) \epsilon$ $X \times X$ is a coupled coincidence point of the pair $(A, S)$, 
whereas $\left(\alpha^{\prime}, \beta^{\prime}\right) \in X \times X$ is a coupled coincidence point of the pair $(B, T)$.

Now we assert that $(\alpha, \beta)=\left(\alpha^{\prime}, \beta^{\prime}\right)$, that is, $\alpha=\alpha^{\prime}$ and $\beta=\beta^{\prime}$. Since $*$ is a $t$-norm of $\mathrm{H}$-type, for any $\lambda>0$, there exists an $\mu>0$ such that

$$
\underbrace{(1-\mu) *(1-\mu) * \cdots *(1-\mu)}_{p} \geq 1-\lambda,
$$

for all $p \in \mathbb{N}$.

Since $M(x, y, \cdot)$ is continuous and $\lim _{t \rightarrow \infty} M(x, y, t)=1$ for all $x, y \in X$, there exists $t_{0}>0$ such that $M\left(\alpha, \alpha^{\prime}, t_{0}\right) \geq$ $1-\mu$ and $M\left(\beta, \beta^{\prime}, t_{0}\right) \geq 1-\mu$.

On the other hand, since $\phi \in \Phi$, by condition $(\phi-3)$, we have $\sum_{n=1}^{\infty} \phi^{n}\left(t_{0}\right)<\infty$. Then for any $t>0$, there exists $n_{0} \in \mathbb{N}$ such that $t>\sum_{p=n_{0}}^{\infty} \phi^{p}\left(t_{0}\right)$. On using inequality (15) with $x=$ $x_{n}, y=y_{n}, u=x_{n}^{\prime}$, and $v=y_{n}^{\prime}$, we have

$$
\begin{aligned}
& M\left(A\left(x_{n}, y_{n}\right), B\left(x_{n}^{\prime}, y_{n}^{\prime}\right), \phi\left(t_{0}\right)\right) \\
& \quad \geq M\left(S x_{n}, T x_{n}^{\prime}, t_{0}\right) * M\left(S y_{n}, T y_{n}^{\prime}, t_{0}\right) .
\end{aligned}
$$

Letting $n \rightarrow \infty$, we get

$$
M\left(\alpha, \alpha^{\prime}, \phi\left(t_{0}\right)\right) \geq M\left(\alpha, \alpha^{\prime}, t_{0}\right) * M\left(\beta, \beta^{\prime}, t_{0}\right) .
$$

Again using inequality (15) with $x=y_{n}, y=x_{n}, u=y_{n}^{\prime}$, and $v=x_{n}^{\prime}$, we have

$$
\begin{aligned}
& M\left(A\left(y_{n}, x_{n}\right), B\left(y_{n}^{\prime}, x_{n}^{\prime}\right), \phi\left(t_{0}\right)\right) \\
& \quad \geq M\left(S y_{n}, T y_{n}^{\prime}, t_{0}\right) * M\left(S x_{n}, T x_{n}^{\prime}, t_{0}\right) .
\end{aligned}
$$

Letting $n \rightarrow \infty$, we get

$$
M\left(\beta, \beta^{\prime}, \phi\left(t_{0}\right)\right) \geq M\left(\beta, \beta^{\prime}, t_{0}\right) * M\left(\alpha, \alpha^{\prime}, t_{0}\right) .
$$

From (22) and (24), we obtain

$$
\begin{aligned}
& M\left(\alpha, \alpha^{\prime}, \phi\left(t_{0}\right)\right) * M\left(\beta, \beta^{\prime}, \phi\left(t_{0}\right)\right) \\
& \quad \geq\left[M\left(\alpha, \alpha^{\prime}, t_{0}\right)\right]^{2} *\left[M\left(\beta, \beta^{\prime}, t_{0}\right)\right]^{2} .
\end{aligned}
$$

In general, for all $n \in \mathbb{N}$, we have

$$
\begin{aligned}
& M\left(\alpha, \alpha^{\prime}, \phi^{n}\left(t_{0}\right)\right) * M\left(\beta, \beta^{\prime}, \phi^{n}\left(t_{0}\right)\right) \\
& \quad \geq\left[M\left(\alpha, \alpha^{\prime}, \phi^{n-1}\left(t_{0}\right)\right)\right]^{2} *\left[M\left(\beta, \beta^{\prime}, \phi^{n-1}\left(t_{0}\right)\right)\right]^{2} \\
& \quad \geq\left[M\left(\alpha, \alpha^{\prime}, t_{0}\right)\right]^{2^{n}} *\left[M\left(\beta, \beta^{\prime}, t_{0}\right)\right]^{2^{n}} .
\end{aligned}
$$

Then, we have

$$
\begin{aligned}
& M\left(\alpha, \alpha^{\prime}, t\right) * M\left(\beta, \beta^{\prime}, t\right) \\
& \geq {\left[M\left(\alpha, \alpha^{\prime}, \sum_{p=n_{0}}^{\infty} \phi^{p}\left(t_{0}\right)\right)\right] } \\
& *\left[M\left(\beta, \beta^{\prime}, \sum_{p=n_{0}}^{\infty} \phi^{p}\left(t_{0}\right)\right)\right] \\
& \geq {\left[M\left(\alpha, \alpha^{\prime}, \phi^{n_{0}}\left(t_{0}\right)\right)\right] } \\
& *\left[M\left(\beta, \beta^{\prime}, \phi^{n_{0}}\left(t_{0}\right)\right)\right] \\
& \geq {\left[M\left(\alpha, \alpha^{\prime}, t_{0}\right)\right]^{2^{n_{0}}} *\left[M\left(\beta, \beta^{\prime}, t_{0}\right)\right]^{2^{n_{0}}} } \\
& \geq \underbrace{(1-\mu) *(1-\mu) * \cdots(1-\mu)}_{2^{2 n_{0}}} \\
& \geq 1-\lambda .
\end{aligned}
$$

So for any $\lambda>0$, we have

$$
M\left(\alpha, \alpha^{\prime}, t\right) * M\left(\beta, \beta^{\prime}, t\right) \geq 1-\lambda
$$

for all $t>0$, and so $\alpha=\alpha^{\prime}$ and $\beta=\beta^{\prime}$. Therefore we have

$$
\begin{array}{ll}
A(\alpha, \beta)=S \alpha, & A(\beta, \alpha)=S \beta, \\
B(\alpha, \beta)=T \alpha, & B(\beta, \alpha)=T \beta .
\end{array}
$$

Next, we show that $S \alpha=T \alpha$ and $S \beta=T \beta$. Since $*$ is a $t$-norm of $\mathrm{H}$-type, for any $\lambda>0$, there exists an $\mu>0$ such that

$$
\underbrace{(1-\mu) *(1-\mu) * \cdots *(1-\mu)}_{p} \geq 1-\lambda,
$$

for all $p \in \mathbb{N}$.

Since $M(x, y, \cdot)$ is continuous and $\lim _{t \rightarrow+\infty} M(x, y, t)=$ 1 for all $x, y \in X$, there exists $t_{0}>0$ such that $M\left(S \alpha, T \alpha, t_{0}\right) \geq$ $1-\mu$ and $M\left(S \beta, T \beta, t_{0}\right) \geq 1-\mu$.

Since $\phi \in \Phi$, by condition $(\phi-3)$, we have $\sum_{n=1}^{\infty} \phi^{n}\left(t_{0}\right)<$ $\infty$. Then for any $t>0$, there exists $n_{0} \in \mathbb{N}$ such that $t>$ $\sum_{p=n_{0}}^{\infty} \phi^{p}\left(t_{0}\right)$. On using inequality (15) with $x=u=\alpha, y=$ $v=\beta$, we have

$$
\begin{aligned}
& M\left(A(\alpha, \beta), B(\alpha, \beta), \phi\left(t_{0}\right)\right) \\
& \quad \geq M\left(S \alpha, T \alpha, t_{0}\right) * M\left(S \beta, T \beta, t_{0}\right),
\end{aligned}
$$

and so

$$
M\left(S \alpha, T \alpha, \phi\left(t_{0}\right)\right) \geq M\left(S \alpha, T \alpha, t_{0}\right) * M\left(S \beta, T \beta, t_{0}\right) .
$$

Similarly, we can obtain

$$
M\left(S \beta, T \beta, \phi\left(t_{0}\right)\right) \geq M\left(S \beta, T \beta, t_{0}\right) * M\left(S \alpha, T \alpha, t_{0}\right) .
$$

From (32) and (33), we have

$$
\begin{aligned}
& M\left(S \alpha, T \alpha, \phi\left(t_{0}\right)\right) * M\left(S \beta, T \beta, \phi\left(t_{0}\right)\right) \\
& \quad \geq\left[M\left(S \alpha, T \alpha, t_{0}\right)\right]^{2} *\left[M\left(S \beta, T \beta, t_{0}\right)\right]^{2} .
\end{aligned}
$$


In general, for all $n \in \mathbb{N}$, we get

$$
\begin{aligned}
M(S \alpha & \left., T \alpha, \phi^{n}\left(t_{0}\right)\right) * M\left(S \beta, T \beta, \phi^{n}\left(t_{0}\right)\right) \\
& \geq\left[M\left(S \alpha, T \alpha, \phi^{n-1}\left(t_{0}\right)\right)\right]^{2} *\left[M\left(S \beta, T \beta, \phi^{n-1}\left(t_{0}\right)\right)\right]^{2} \\
& \geq\left[M\left(S \alpha, T \alpha, t_{0}\right)\right]^{2^{n}} *\left[M\left(S \beta, T \beta, t_{0}\right)\right]^{2^{n}} .
\end{aligned}
$$

Then, we have

$$
\begin{aligned}
& M(S \alpha, T \alpha, t) * M(S \beta, T \beta, t) \\
& \geq {\left[M\left(S \alpha, T \alpha, \sum_{p=n_{0}}^{\infty} \phi^{p}\left(t_{0}\right)\right)\right] } \\
& *\left[M\left(S \beta, T \beta, \sum_{p=n_{0}}^{\infty} \phi^{p}\left(t_{0}\right)\right)\right] \\
& \geq {\left[M\left(S \alpha, T \alpha, \phi^{n_{0}}\left(t_{0}\right)\right)\right] } \\
& *\left[M\left(S \beta, T \beta, \phi^{n_{0}}\left(t_{0}\right)\right)\right] \\
& \geq {\left[M\left(S \alpha, T \alpha, t_{0}\right)\right]^{2^{n_{0}}} } \\
& *\left[M\left(S \beta, T \beta, t_{0}\right)\right]^{2^{n_{0}}} \\
& \geq \underbrace{(1-\mu) *(1-\mu) * \cdots *(1-\mu)}_{2^{2 n_{0}}} \\
& \geq 1-\lambda .
\end{aligned}
$$

So for any $\lambda>0$, we obtain

$$
M(S \alpha, T \alpha, t) * M(S \beta, T \beta, t) \geq 1-\lambda,
$$

for all $t>0$, and hence $S \alpha=T \alpha$ and $S \beta=T \beta$. Therefore

$$
\begin{aligned}
& S \alpha=T \alpha=A(\alpha, \beta)=B(\alpha, \beta), \\
& S \beta=T \beta=A(\beta, \alpha)=B(\beta, \alpha) .
\end{aligned}
$$

Now we show that $S \alpha=\alpha$ and $S \beta=\beta$. Since $*$ is a $t$-norm of $\mathrm{H}$-type, for any $\lambda>0$, there exists an $\mu>0$ such that

$$
\underbrace{(1-\mu) *(1-\mu) * \cdots *(1-\mu)}_{p} \geq 1-\lambda,
$$

for all $p \in \mathbb{N}$.

Since $M(x, y, \cdot)$ is continuous and $\lim _{t \rightarrow+\infty} M(x, y, t)=$ 1 for all $x, y \in X$, there exists $t_{0}>0$ such that $M\left(S \alpha, \alpha, t_{0}\right) \geq$ $1-\mu$ and $M\left(S \beta, \beta, t_{0}\right) \geq 1-\mu$.

On the other hand, since $\phi \in \Phi$, by condition $(\phi-3)$ we have $\sum_{n=1}^{\infty} \phi^{n}\left(t_{0}\right)<\infty$. Then for any $t>0$, there exists $n_{0} \in \mathbb{N}$ such that $t>\sum_{p=n_{0}}^{\infty} \phi^{p}\left(t_{0}\right)$. On using inequality (15) with $x=$ $\alpha, y=\beta, u=x_{n}^{\prime}, v=y_{n}^{\prime}$, we have

$$
\begin{aligned}
& M\left(A(\alpha, \beta), B\left(x_{n}^{\prime}, y_{n}^{\prime}\right), \phi\left(t_{0}\right)\right) \\
& \quad \geq M\left(S \alpha, T x_{n}^{\prime}, t_{0}\right) * M\left(S \beta, T y_{n}^{\prime}, t_{0}\right) .
\end{aligned}
$$

Letting $n \rightarrow \infty$, we obtain

$$
M\left(S \alpha, \alpha, \phi\left(t_{0}\right)\right) \geq M\left(S \alpha, \alpha, t_{0}\right) * M\left(S \beta, \beta, t_{0}\right) .
$$

Similarly, we can get

$$
M\left(S \beta, \beta, \phi\left(t_{0}\right)\right) \geq M\left(S \beta, \beta, t_{0}\right) * M\left(S \alpha, \alpha, t_{0}\right) .
$$

Consequently, from (41) and (42), we have

$$
\begin{aligned}
& M\left(S \alpha, \alpha, \phi\left(t_{0}\right)\right) * M\left(S \beta, \beta, \phi\left(t_{0}\right)\right) \\
& \quad \geq\left[M\left(S \alpha, \alpha, t_{0}\right)\right]^{2} *\left[M\left(S \beta, \beta, t_{0}\right)\right]^{2} .
\end{aligned}
$$

In general, for all $n \in \mathbb{N}$, we get

$$
\begin{aligned}
& M\left(S \alpha, \alpha, \phi^{n}\left(t_{0}\right)\right) * M\left(S \beta, \beta, \phi^{n}\left(t_{0}\right)\right) \\
& \quad \geq\left[M\left(S \alpha, \alpha, \phi^{n-1}\left(t_{0}\right)\right)\right]^{2} *\left[M\left(S \beta, \beta, \phi^{n-1}\left(t_{0}\right)\right)\right]^{2} \\
& \quad \geq\left[M\left(S \alpha, \alpha, t_{0}\right)\right]^{2^{n}} *\left[M\left(S \beta, \beta, t_{0}\right)\right]^{2^{n}} .
\end{aligned}
$$

Then, we have

$$
\begin{aligned}
& M(S \alpha, \alpha, t) * M(S \beta, \beta, t) \\
& \geq {\left[M\left(S \alpha, \alpha, \sum_{p=n_{0}}^{\infty} \phi^{p}\left(t_{0}\right)\right)\right] } \\
& *\left[M\left(S \beta, \beta, \sum_{p=n_{0}}^{\infty} \phi^{p}\left(t_{0}\right)\right)\right] \\
& \geq {\left[M\left(S \alpha, \alpha, \phi^{n_{0}}\left(t_{0}\right)\right)\right] } \\
& *\left[M\left(S \beta, \beta, \phi^{n_{0}}\left(t_{0}\right)\right)\right] \\
& \geq {\left[M\left(S \alpha, \alpha, t_{0}\right)\right]^{2^{n_{0}}} } \\
& *\left[M\left(S \beta, \beta, t_{0}\right)\right]^{2^{n_{0}}} \\
& \geq \underbrace{(1-\mu) *(1-\mu) * \cdots *(1-\mu)}_{2^{2 n_{0}}} \\
& \geq 1-\lambda .
\end{aligned}
$$

Therefore for any $\lambda>0$, we obtain

$$
M(S \alpha, \alpha, t) * M(S \beta, \beta, t) \geq 1-\lambda,
$$

for all $t>0$ and so $S \alpha=\alpha$ and $S \beta=\beta$. Thus

$$
\begin{aligned}
& \alpha=S \alpha=T \alpha=A(\alpha, \beta)=B(\alpha, \beta), \\
& \beta=S \beta=T \beta=A(\beta, \alpha)=B(\beta, \alpha) .
\end{aligned}
$$

Finally, we assert that $\alpha=\beta$. Since $*$ is a $t$-norm of $\mathrm{H}$-type, for any $\lambda>0$, there exists an $\mu>0$ such that

$$
\underbrace{(1-\mu) *(1-\mu) * \cdots *(1-\mu)}_{p} \geq 1-\lambda
$$

for all $p \in \mathbb{N}$. 
Since $M(x, y, \cdot)$ is continuous and $\lim _{t \rightarrow+\infty} M(x, y, t)=$ 1 for all $x, y \in X$, there exists $t_{0}>0$ such that $M\left(\alpha, \beta, t_{0}\right) \geq$ $1-\mu$.

Also, since $\phi \in \Phi$, by condition $(\phi-3)$, we have $\sum_{n=1}^{\infty} \phi^{n}\left(t_{0}\right)<\infty$. Then for any $t>0$, there exists $n_{0} \in \mathbb{N}$ such that $t>\sum_{p=n_{0}}^{\infty} \phi^{p}\left(t_{0}\right)$. On using inequality (15) with $x=v=\alpha, y=u=\beta$, we have

$$
\begin{aligned}
& M\left(A(\alpha, \beta), B(\beta, \alpha), \phi\left(t_{0}\right)\right) \\
& \quad \geq M\left(S \alpha, T \beta, t_{0}\right) * M\left(S \beta, T \alpha, t_{0}\right),
\end{aligned}
$$

and so

$$
M\left(\alpha, \beta, \phi\left(t_{0}\right)\right) \geq M\left(\alpha, \beta, t_{0}\right) * M\left(\beta, \alpha, t_{0}\right) .
$$

Thus we have

$$
\begin{aligned}
M(\alpha, \beta, t) & \geq M\left(\alpha, \beta, \sum_{p=n_{0}}^{\infty} \phi^{p}\left(t_{0}\right)\right) \\
& \geq M\left(\alpha, \beta, \phi^{n_{0}}\left(t_{0}\right)\right) \\
& \geq\left[M\left(\alpha, \beta, t_{0}\right)\right]^{2^{n_{0}}} *\left[M\left(\beta, \alpha, t_{0}\right)\right]^{2^{n_{0}}} \\
& \geq \underbrace{(1-\mu) *(1-\mu) * \cdots *(1-\mu)}_{2^{2 n_{0}}} \\
& \geq 1-\lambda,
\end{aligned}
$$

which implies that $\alpha=\beta$. Therefore, we proved that there exists $\alpha$ in $X$ such that

$$
\alpha=S \alpha=T \alpha=A(\alpha, \alpha)=B(\alpha, \alpha) \text {. }
$$

The uniqueness of such a point follows immediately from inequality (15) and so we omit the details.

Remark 15. The conclusion of Theorem 14 remains true if we substitute condition (a) with the following condition:

$\left(\mathrm{a}^{\prime}\right)$ the pairs $(A, S)$ and $(B, T)$ are subcompatible and reciprocally continuous.

From Theorem 14, taking $A=B$ and $S=T$, we deduce the following natural result.

Corollary 16. Let $(X, M, *)$ be a fuzzy metric space, where * is a continuous $t$-norm of H-type such that $M(x, y, t) \rightarrow 1$ as $t \rightarrow \infty$, for all $x, y \in X$. Let $A: X \times X \rightarrow X$ and $S: X \rightarrow$ $X$ be compatible and subsequentially continuous (alternately subcompatible and reciprocally continuous) mappings such that

$$
\begin{aligned}
& M(A(x, y), A(u, v), \phi(t)) \\
& \quad \geq M(S x, S u, t) * M(S y, S v, t),
\end{aligned}
$$

for all $x, y, u, v \in X, \phi \in \Phi$ and $t>0$. Then there exists a unique point $\alpha$ in $X$ such that $\alpha=S \alpha=A(\alpha, \alpha)$.

Next, we illustrate our results providing the following examples.
Example 17. Let $X=[0,+\infty), a * b=a b$ for all $a, b \in[0,1]$ and $\psi(s)=s /(s+1)$ for all $s \in \mathbb{R}^{+}$. Then $(X, M, *)$ is a fuzzy metric space, where

$$
M(x, y, t)=[\psi(t)]^{|x-y|},
$$

for all $x, y \in X$ and $t>0$. Let $\phi(s)=s / 2$, and let the mappings $A: X \times X \rightarrow X, S: X \rightarrow X$ be defined as

$$
A(x, y)= \begin{cases}3 x+3 y-5, & \text { if } x, y \in(1, \infty) \\ \frac{x+y}{6}, & \text { otherwise }\end{cases}
$$

$$
S(x)= \begin{cases}3 x-2, & \text { if } x \in(1, \infty), \\ \frac{x}{6}, & \text { if } x \in[0,1] .\end{cases}
$$

In view of Definition 10, to prove compatibility, we have only to consider sequences $\left\{x_{n}\right\}$ and $\left\{y_{n}\right\}$ converging to zero from the right. In such case we have

$$
\begin{aligned}
& \lim _{n \rightarrow \infty} A\left(x_{n}, y_{n}\right)=0=\lim _{n \rightarrow \infty} S\left(x_{n}\right), \\
& \lim _{n \rightarrow \infty} A\left(y_{n}, x_{n}\right)=0=\lim _{n \rightarrow \infty} S\left(y_{n}\right) .
\end{aligned}
$$

Next, we get

$$
\begin{gathered}
\lim _{n \rightarrow \infty} A\left(S x_{n}, S y_{n}\right)=0=A(0,0), \\
\lim _{n \rightarrow \infty} S A\left(x_{n}, y_{n}\right)=0=S(0), \\
\lim _{n \rightarrow \infty} A\left(S y_{n}, S x_{n}\right)=0=A(0,0), \\
\lim _{n \rightarrow \infty} S A\left(y_{n}, x_{n}\right)=0=S(0) .
\end{gathered}
$$

Consequently

$$
\begin{aligned}
& \lim _{n \rightarrow \infty} M\left(A\left(S x_{n} S y_{n}\right), S A\left(x_{n}, y_{n}\right), t\right)=1, \\
& \lim _{n \rightarrow \infty} M\left(A\left(S y_{n} S x_{n}\right), S A\left(y_{n}, x_{n}\right), t\right)=1,
\end{aligned}
$$

for all $t>0$.

On the other hand, to prove subsequential continuity, in view of Definition 12, we have only to consider sequences $\left\{x_{n}\right\}$ and $\left\{y_{n}\right\}$ converging to one from the right. In such case we have

$$
\begin{aligned}
& \lim _{n \rightarrow \infty} A\left(x_{n}, y_{n}\right)=1=\lim _{n \rightarrow \infty} S\left(x_{n}\right), \\
& \lim _{n \rightarrow \infty} A\left(y_{n}, x_{n}\right)=1=\lim _{n \rightarrow \infty} S\left(y_{n}\right) .
\end{aligned}
$$

Also, note that, for the same sequences, we get

$$
\begin{gathered}
\lim _{n \rightarrow \infty} A\left(S x_{n}, S y_{n}\right)=1 \neq A(1,1), \\
\lim _{n \rightarrow \infty} S A\left(x_{n}, y_{n}\right)=1 \neq S(1), \\
\lim _{n \rightarrow \infty} A\left(S y_{n}, S x_{n}\right)=1 \neq A(1,1), \\
\lim _{n \rightarrow \infty} S A\left(y_{n}, x_{n}\right)=1 \neq S(1),
\end{gathered}
$$


but

$$
\begin{aligned}
& \lim _{n \rightarrow \infty} M\left(A\left(S x_{n}, S y_{n}\right), S A\left(x_{n}, y_{n}\right), t\right)=1, \\
& \lim _{n \rightarrow \infty} M\left(A\left(S y_{n}, S x_{n}\right), S A\left(y_{n}, x_{n}\right), t\right)=1 .
\end{aligned}
$$

Thus, the mappings $A$ and $S$ are compatible as well as subsequentially continuous but not reciprocally continuous. Next, by a routine calculation, one can verify that condition (53) holds true. For instance, for all $t>0$ and $x, y, u, v \in$ $[0,1]$, we have

$$
\begin{aligned}
M(A & (x, y), A(u, v), \phi(t)) \\
& =M\left(A(x, y), A(u, v),\left(\frac{t}{2}\right)\right) \\
& =\left[\psi\left(\frac{t}{2}\right)\right]^{|x-u+y-v| / 6} \\
& \geq\left[\psi\left(\frac{t}{2}\right)\right]^{|x-u| / 6} \cdot\left[\psi\left(\frac{t}{2}\right)\right]^{|y-v| / 6} \\
& =\left[\frac{t}{t+2}\right]^{|x-u| / 6} \cdot\left[\frac{t}{t+2}\right]^{|y-v| / 6} \\
& \geq\left[\frac{t}{t+1}\right]^{|x-u| / 6} \cdot\left[\frac{t}{t+1}\right]^{|y-v| / 6} \\
& =[\psi(t)]^{|x-u| / 6} \cdot[\psi(t)]^{|y-v| / 6} \\
& =M(S x, S u, t) \cdot M(S y, S v, t) \\
& =M(S x, S u, t) * M(S y, S v, t) .
\end{aligned}
$$

Therefore, all the conditions of Corollary 16 are satisfied and $(0,0)$ is the unique common fixed point of the pair $(A, S)$. It is noted that this example cannot be covered by those fixed point theorems which involve compatibility and reciprocal continuity both.

Example 18. In the setting of Example 17 (besides retaining the rest), let $X=(-\infty, \infty)$, and let the mappings $A: X \times$ $X \rightarrow X, S: X \rightarrow X$ be defined as

$$
\begin{aligned}
A(x, y)= \begin{cases}\frac{x+y}{4}, & \text { if } x, y \in(-\infty, 1), \\
3 x+3 y-5, & \text { if } x, y \in[1, \infty), \\
\frac{x-y}{4}, & \text { if } x \in(-\infty, 1), y \in[1, \infty),\end{cases} \\
S(x)= \begin{cases}x+1, & \text { if } x \in(-\infty, 1), \\
3 x-2, & \text { if } x \in[1, \infty) .\end{cases}
\end{aligned}
$$

In view of Definitions 11 and 13, to prove reciprocal continuity and subcompatibility, we have only to consider sequences $\left\{x_{n}\right\}$ and $\left\{y_{n}\right\}$ converging to one from the right. For such sequences, we get

$$
\begin{aligned}
& \lim _{n \rightarrow \infty} A\left(x_{n}, y_{n}\right)=1=\lim _{n \rightarrow \infty} S\left(x_{n}\right), \\
& \lim _{n \rightarrow \infty} A\left(y_{n}, x_{n}\right)=1=\lim _{n \rightarrow \infty} S\left(y_{n}\right) .
\end{aligned}
$$

Also, we deduce that

$$
\begin{gathered}
\lim _{n \rightarrow \infty} A\left(S x_{n}, S y_{n}\right)=1=A(1,1), \\
\lim _{n \rightarrow \infty} S A\left(x_{n}, y_{n}\right)=1=S(1), \\
\lim _{n \rightarrow \infty} A\left(S y_{n}, S x_{n}\right)=1=A(1,1), \\
\lim _{n \rightarrow \infty} S A\left(y_{n}, x_{n}\right)=1=S(1) .
\end{gathered}
$$

Therefore, we have

$$
\begin{aligned}
& \lim _{n \rightarrow \infty} M\left(A\left(S x_{n}, S y_{n}\right), S A\left(x_{n}, y_{n}\right), t\right)=1, \\
& \lim _{n \rightarrow \infty} M\left(A\left(S y_{n}, S x_{n}\right), S A\left(y_{n}, x_{n}\right), t\right)=1,
\end{aligned}
$$

for all $t>0$. Finally, to show that the mappings $A$ and $S$ are not compatible, it suffices to consider the particular sequences $\left\{x_{n}\right\}=\{1 / n-2\}_{n \in \mathbb{N}}$ and $\left\{y_{n}\right\}=\{1 / 3 n-2\}_{n \in \mathbb{N}}$ in $X$. In fact, in such case, we have

$$
\begin{aligned}
& \lim _{n \rightarrow \infty} A\left(x_{n}, y_{n}\right)=-1=\lim _{n \rightarrow \infty} S\left(x_{n}\right), \\
& \lim _{n \rightarrow \infty} A\left(y_{n}, x_{n}\right)=-1=\lim _{n \rightarrow \infty} S\left(y_{n}\right) .
\end{aligned}
$$

Next, we deduce that

$$
\begin{gathered}
\lim _{n \rightarrow \infty} A\left(S x_{n}, S y_{n}\right)=-\frac{1}{2}=A(-1,-1), \\
\lim _{n \rightarrow \infty} S A\left(x_{n}, y_{n}\right)=0=S(-1), \\
\lim _{n \rightarrow \infty} A\left(S y_{n}, S x_{n}\right)=-\frac{1}{2}=A(-1,-1), \\
\lim _{n \rightarrow \infty} S A\left(y_{n}, x_{n}\right)=0=S(-1) .
\end{gathered}
$$

Consequently, we obtain

$$
\begin{aligned}
& \lim _{n \rightarrow \infty} M\left(A\left(S x_{n}, S y_{n}\right), S A\left(x_{n}, y_{n}\right), t\right) \neq 1, \\
& \lim _{n \rightarrow \infty} M\left(A\left(S y_{n}, S x_{n}\right), S A\left(y_{n}, x_{n}\right), t\right) \neq 1,
\end{aligned}
$$

for all $t>0$. Thus, the mappings $A$ and $S$ are reciprocally continuous as well as subcompatible but not compatible. Next, by a routine calculation, one can verify that condition 
(53) holds true. For instance, for all $t>0$ and $x, y, u, v \in$ $[1, \infty)$, we have

$$
\begin{aligned}
M(A & (x, y), A(u, v), \phi(t)) \\
& =M\left(A(x, y), A(u, v),\left(\frac{t}{2}\right)\right) \\
& =\left[\psi\left(\frac{t}{2}\right)\right]^{|3 x-3 u+3 y-3 v|} \\
& \geq\left[\psi\left(\frac{t}{2}\right)\right]^{3|x-u|} \cdot\left[\psi\left(\frac{t}{2}\right)\right]^{3|y-v|} \\
& =\left[\frac{t}{t+2}\right]^{3|x-u|} \cdot\left[\frac{t}{t+2}\right]^{3|y-v|} \\
& \geq\left[\frac{t}{t+1}\right]^{3|x-u|} \cdot\left[\frac{t}{t+1}\right]^{3|y-v|} \\
& =[\psi(t)]^{3|x-u|} \cdot[\psi(t)]^{3|y-v|} \\
& =M(S x, S u, t) \cdot M(S y, S v, t) \\
& =M(S x, S u, t) * M(S y, S v, t) .
\end{aligned}
$$

Therefore, all the conditions of Corollary 16 are satisfied, and $(1,1)$ is the unique common fixed point of the pair $(A, S)$. It is also noted that this example cannot be covered by those fixed point theorems which involve compatibility and reciprocal continuity both.

Remark 19. The conclusions of Theorem 14 and Corollary 16 remain true if we assume $\phi(s)=k s$, where $k \in(0,1)$.

\section{Conclusion}

Theorem 14 is proved for two pairs of compatible and subsequentially continuous (alternately subcompatible and reciprocally continuous) mappings in fuzzy metric spaces, wherein conditions on completeness (or closedness) of the underlying space (or subspaces) together with conditions on continuity in respect to anyone of the involved mappings are relaxed. Theorem 14 improves the results of Jain et al. [39, Theorem 3.2, Corollary 3.2, Theorem 3.3, Theorem 3.4, Theorem 4.1] and $\mathrm{Hu}$ [34, Theorem 1]. A natural result is also obtained for a pair of mappings (see Corollary 16). Finally, Examples 17 and 18 are furnished to demonstrate the usefulness of Corollary 16. In view of Remark 19, Theorem 14 and Corollary 16 improve the results of Sedghi et al. [32, Theorem 2.5, Corollary 2.6] and Jain et al. [39, Corollary 3.1].

\section{Acknowledgments}

The authors would like to express their sincere thanks to the editor and reviewer(s) for their valuable suggestions.

\section{References}

[1] L. A. Zadeh, "Fuzzy sets," Information and Computation, vol. 8, pp. 338-353, 1965.
[2] I. Kramosil and J. Michalek, "Fuzzy metric and statistical metric spaces," Kybernetika, vol. 11, no. 5, pp. 336-344, 1975.

[3] M. Grabiec, "Fixed points in fuzzy metric spaces," Fuzzy Sets and Systems, vol. 27, no. 3, pp. 385-389, 1988.

[4] P. Balasubramaniam, S. Muralisankar, and R. P. Pant, "Common fixed points of four mappings in a fuzzy metric space," Journal of Fuzzy Mathematics, vol. 10, no. 2, pp. 379-384, 2002.

[5] Y. J. Cho, "Fixed points in fuzzy metric spaces," Journal of Fuzzy Mathematics, vol. 5, no. 4, pp. 949-962, 1997.

[6] J.-X. Fang, "On fixed point theorems in fuzzy metric spaces," Fuzzy Sets and Systems, vol. 46, no. 1, pp. 107-113, 1992.

[7] A. George and P. Veeramani, "On some results in fuzzy metric spaces," Fuzzy Sets and Systems, vol. 64, no. 3, pp. 395-399, 1994.

[8] P. V. Subrahmanyam, "A common fixed point theorem in fuzzy metric spaces," Information Sciences, vol. 83, no. 3-4, pp. 109-112, 1995.

[9] R. Vasuki, "Common fixed points for R-weakly commuting maps in fuzzy metric spaces," Indian Journal of Pure and Applied Mathematics, vol. 30, no. 4, pp. 419-423, 1999.

[10] S. N. Mishra, N. Sharma, and S. L. Singh, "Common fixed points of maps on fuzzy metric spaces," International Journal of Mathematics and Mathematical Sciences, vol. 17, no. 2, pp. 253258, 1994.

[11] G. Jungck, "Compatible mappings and common fixed points," International Journal of Mathematics and Mathematical Sciences, vol. 9, no. 4, pp. 771-779, 1986.

[12] B. Singh and S. Jain, "Semicompatibility and fixed point theorems in fuzzy metric space using implicit relation," International Journal of Mathematics and Mathematical Sciences, vol. 2005, no. 16, pp. 2617-2629, 2005.

[13] H. Bouhadjera and C. Godet-Thobie, "Common fixed point theorems forpairs of subcompatible maps," http://arxiv.org/ abs/0906.3159.

[14] H. Bouhadjera and C. Godet-Thobie, "Common fixed point theorems for pairs of subcompatible maps," http://arxiv.org/ abs/0906.3159v2.

[15] D. Gopal and M. Imdad, "Some new common fixed point theorems in fuzzy metric spaces," Annali dell'Universita di Ferrara, vol. 57, no. 2, pp. 303-316, 2011.

[16] M. Imdad, J. Ali, and M. Tanveer, "Remarks on some recent metrical common fixed point theorems," Applied Mathematics Letters, vol. 24, no. 7, pp. 1165-1169, 2011.

[17] M. Abbas, I. Altun, and D. Gopal, "Common fixed point theorems for non compatible mappings in fuzzy metric spaces," Bulletin of Mathematical Analysis and Applications, vol. 1, no. 2, pp. 47-56, 2009.

[18] C. di Bari and C. Vetro, "A fixed point theorem for a family of mappings in a fuzzy metric space," Rendiconti del Circolo Matematico di Palermo, vol. 52, no. 2, pp. 315-321, 2003.

[19] S. Chauhan, S. Bhatnagar, and S. Radenović, "Common fixed point theorems for weakly compatible mappings in fuzzy metric spaces," Le Matematiche, vol. 68, no. 1, pp. 87-98, 2013.

[20] M. Imdad and J. Ali, "Some common fixed point theorems in fuzzy metric spaces," Mathematical Communications, vol. 11, no. 2, pp. 153-163, 2006.

[21] S. Kumar and S. Chauhan, "Common fixed point theorems using implicit relation and property (E.A) in fuzzy metric spaces," Annals of Fuzzy Mathematics and Informatics, vol. 5, no. 1, pp. 107-114, 2013. 
[22] D. Miheț, "A Banach contraction theorem in fuzzy metric spaces," Fuzzy Sets and Systems, vol. 144, no. 3, pp. 431-439, 2004.

[23] D. Miheț, "Fixed point theorems in fuzzy metric spaces using property E.A," Nonlinear Analysis, vol. 73, no. 7, pp. 2184-2188, 2010.

[24] D. O'Regan and M. Abbas, "Necessary and sufficient conditions for common fixed point theorems in fuzzy metric space," Demonstratio Mathematica, vol. 42, no. 4, pp. 887-900, 2009.

[25] B. D. Pant and S. Chauhan, "Common fixed point theorems for two pairs of weakly compatible mappings in Menger spaces and fuzzy metric spaces," Scientific Studies and Research, vol. 21, no. 2, pp. 81-96, 2011.

[26] C. Vetro and P. Vetro, "Common fixed points for discontinuous mappings in fuzzy metric spaces," Rendiconti del Circolo Matematico di Palermo, vol. 57, no. 2, pp. 295-303, 2008.

[27] T. G. Bhaskar and V. Lakshmikantham, "Fixed point theorems in partially ordered metric spaces and applications," Nonlinear Analysis, vol. 65, no. 7, pp. 1379-1393, 2006.

[28] V. Lakshmikantham and L. Ćirić, "Coupled fixed point theorems for nonlinear contractions in partially ordered metric spaces," Nonlinear Analysis, vol. 70, no. 12, pp. 4341-4349, 2009.

[29] D. Đorić, Z. Kadelburg, and S. Radenović, "Coupled fixed point results for mappings without mixed monotone property," Applied Mathematics Letters, vol. 25, no. 11, pp. 1803-1808, 2012.

[30] Z. Golubović, Z. Kadelburg, and S. Radenović, "Coupled coincidence points of mappings in ordered partial metric spaces," Abstract and Applied Analysis, vol. 2012, Article ID 192581, 18 pages, 2012.

[31] Z. Kadelburg and S. Radenović, "Coupled fixed point results under TVS-cone metric and $w$-cone-distance," Advances in Fixed Point Theory, vol. 2, no. 1, pp. 29-46, 2012.

[32] S. Sedghi, I. Altun, and N. Shobe, "Coupled fixed point theorems for contractions in fuzzy metric spaces," Nonlinear Analysis, vol. 72, no. 3-4, pp. 1298-1304, 2010.

[33] J.-X. Fang, "Common fixed point theorems of compatible and weakly compatible maps in Menger spaces," Nonlinear Analysis, vol. 71, no. 5-6, pp. 1833-1843, 2009.

[34] X.-Q. Hu, "Common coupled fixed point theorems for contractive mappings in fuzzy metric spaces," Fixed Point Theory and Applications, vol. 2011, Article ID 363716, 14 pages, 2011.

[35] X.-H. Zhu and J.-Z. Xiao, "Note on "Coupled fixed point theorems for contractions in fuzzy metric spaces'”, Nonlinear Analysis, vol. 74, no. 16, pp. 5475-5479, 2011.

[36] B. Schweizer and A. Sklar, Probabilistic Metric Spaces, NorthHolland Series in Probability and Applied Mathematics, NorthHolland, New York, NY, USA, 1983.

[37] O. Hadžić and E. Pap, Fixed Point Theory in Probabilistic Metric Spaces, vol. 536 of Mathematics and Its Applications, Kluwer Academic, Dodrecht, The Netherlands, 2001.

[38] R. P. Pant and R. K. Bisht, "Common fixed point theorems under a new continuity condition," Annali dell'Universitá di Ferrara, vol. 58, no. 1, pp. 127-141, 2012.

[39] M. Jain, S. Kumar, and R. Chugh, "Coupled fixed point theorems for weak compatible mappings in fuzzy metric spaces," Annals of Fuzzy Mathematics and Informatics, vol. 5, no. 2, pp. 321-336, 2013. 

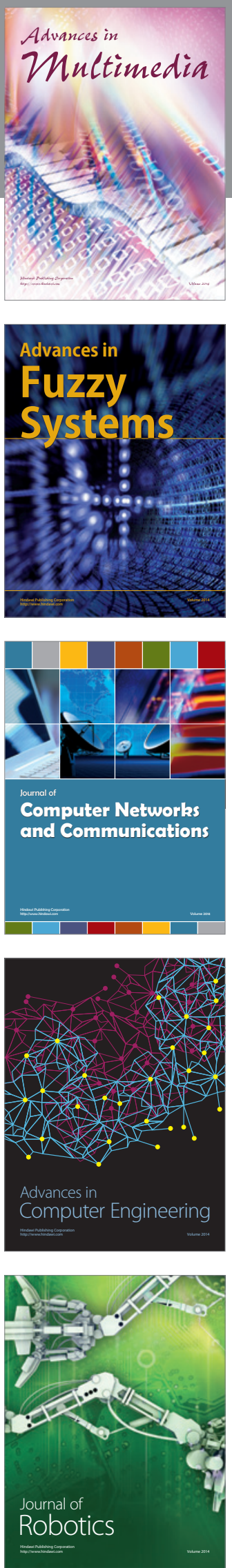

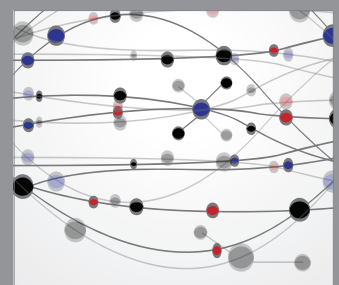

The Scientific World Journal
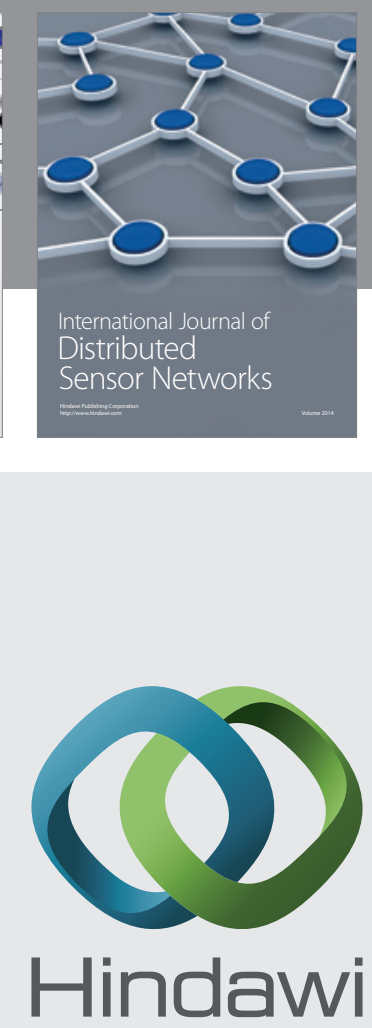

Submit your manuscripts at

http://www.hindawi.com
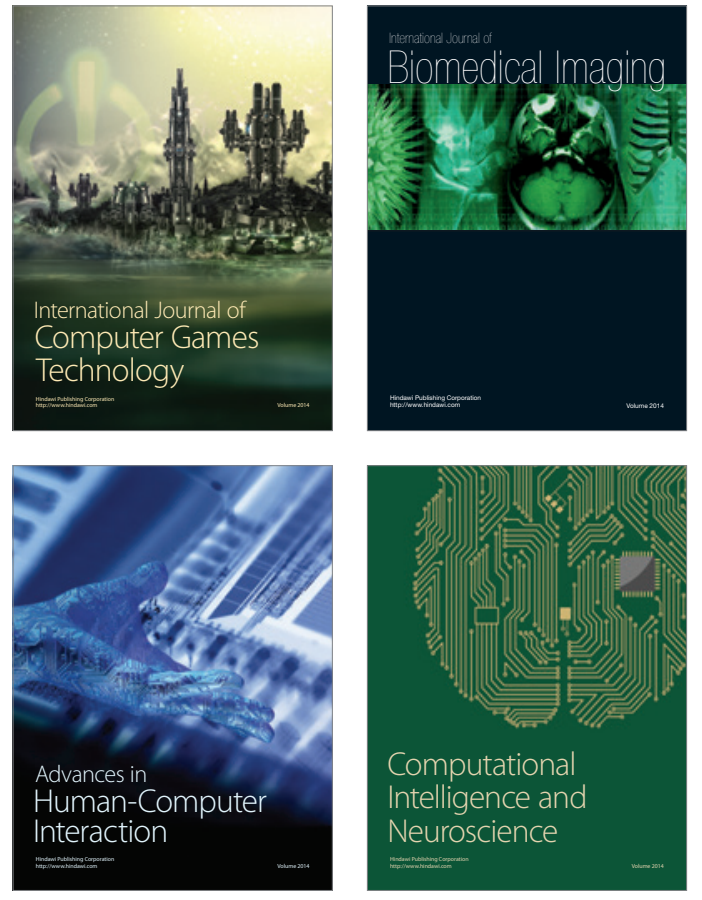
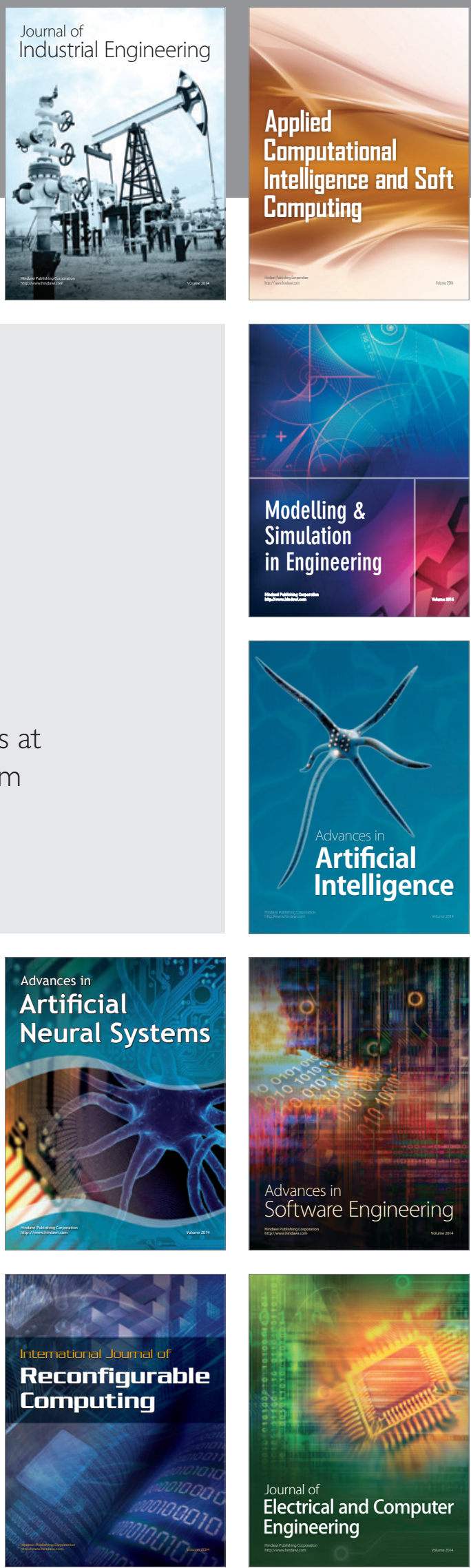